\title{
The Sound of Gravity
}

\section{Thomas Dent* for the LIGO Scientific Collaboration and the Virgo Collaboration}

Max Planck Institute for Gravitational Physics (Albert Einstein Institute)

Callinstraße 38, D-30167 Hannover

E-mail: thomas.denteaei.mpg.de

Since its prediction by Einstein a century ago as a consequence of the general theory of relativity, gravitational radiation has posed a daunting technical challenge, requiring enormously accurate differential length measurements for detection. The first observation of gravitational waves of astrophysical origin was made by the recently upgraded Advanced LIGO [1] detectors in September 2015 [2, 3, 4, 5, 6], the source being the merger of two black holes, each more massive than any previously observed in a stellar system [7]. From data gathered up to January 2016, a second detection [8] and a likely third candidate signal began to reveal a population of binary black hole mergers [9] occurring at a rate close to plausible maximum estimates for standard formation channels $[10,11]$. Tests of the strong-field behaviour of gravity using these events showed no deviations from the predictions of general relativity $[12,9]$. After this spectacular beginning, the next few years will bring the operation of a global network with the Virgo [13] and KAGRA [14] detectors and the possible detection of merging binary systems containing neutron stars which may yield electromagnetic [15] and neutrino [16] signatures.

Neutrino Oscillation Workshop

4 - 11 September, 2016

Otranto (Lecce, Italy)

\footnotetext{
* Speaker.
} 


\section{References}

[1] J. Aasi et al. [LIGO Scientific Collaboration], Class. Quant. Grav. 32 (2015) 074001 [arXiv:1411.4547 [gr-qc]].

[2] B. P. Abbott et al. [LIGO Scientific and Virgo Collaborations], Phys. Rev. Lett. 116 (2016) no.6, 061102 [arXiv:1602.03837 [gr-qc]].

[3] B. P. Abbott et al. [LIGO Scientific and Virgo Collaborations], Phys. Rev. Lett. 116 (2016) no.13, 131103 [arXiv:1602.03838 [gr-qc]].

[4] B. P. Abbott et al. [LIGO Scientific and Virgo Collaborations], Phys. Rev. D 93 (2016) no.12, 122003 [arXiv:1602.03839 [gr-qc]].

[5] B. P. Abbott et al. [LIGO Scientific and Virgo Collaborations], Phys. Rev. D 93 (2016) no.12, 122004 [arXiv:1602.03843 [gr-qc]].

[6] B. P. Abbott et al. [LIGO Scientific and Virgo Collaborations], Class. Quant. Grav. 33 (2016) no.13, 134001 [arXiv:1602.03844 [gr-qc]]; B. P. Abbott et al. [LIGO Scientific Collaboration], arXiv:1602.03845 [gr-qc].

[7] B. P. Abbott et al. [LIGO Scientific and Virgo Collaborations], Phys. Rev. Lett. 116 (2016) no.24, 241102 [arXiv:1602.03840 [gr-qc]].

[8] B. P. Abbott et al. [LIGO Scientific and Virgo Collaborations], Phys. Rev. Lett. 116 (2016) no.24, 241103 [arXiv:1606.04855 [gr-qc]].

[9] B. P. Abbott et al. [LIGO Scientific and Virgo Collaborations], Phys. Rev. X 6 (2016) no.4, 041015 [arXiv:1606.04856 [gr-qc]].

[10] B. P. Abbott et al. [LIGO Scientific and Virgo Collaborations], Astrophys. J. 833 (2016) 1 [arXiv:1602.03842 [astro-ph.HE]]; Astrophys. J. Suppl. 227 (2016) no.2, 14 [arXiv:1606.03939 [astro-ph.HE]].

[11] B. P. Abbott et al. [LIGO Scientific and Virgo Collaborations], Astrophys. J. 818 (2016) no.2, L22 [arXiv:1602.03846 [astro-ph.HE]].

[12] B. P. Abbott et al. [LIGO Scientific and Virgo Collaborations], Phys. Rev. Lett. 116 (2016) no.22, 221101 [arXiv:1602.03841 [gr-qc]].

[13] F. Acernese et al. [VIRGO Collaboration], Class. Quant. Grav. 32 (2015) no.2, 024001 [arXiv:1408.3978 [gr-qc]].

[14] Y. Aso et al. [KAGRA Collaboration], Phys. Rev. D 88 (2013) no.4, 043007 [arXiv:1306.6747 [gr-qc]].

[15] B. P. Abbott et al. [LIGO Scientific and Virgo Collaborations], Living Rev. Rel. 19 (2016) 1 [arXiv:1304.0670 [gr-qc]].

[16] S. Ando et al., Rev. Mod. Phys. 85 (2013) no.4, 1401 [arXiv:1203.5192 [astro-ph.HE]]. 